\title{
Fluctuación poblacional de trips (Frankliniella cf. gardeniae) en cultivos de mango en Tolima, Colombia
}

\author{
Population fluctuation of thrips (Frankliniella cf. gardeniae) in mango crops in Tolima, Colombia
}

\author{
PAOLA VANESSA SIERRA-BAQUERO ${ }^{1}$, EDGAR HERNEY VARÓN-DEVIA² ${ }^{2}$ LUCIMAR GOMES-DÍAS ${ }^{3}$ \\ y CAMILO IGNACIO JARAMILLO-BARRIOS ${ }^{4}$
}

\begin{abstract}
Resumen: El mango es una de las frutas tropicales con mayor producción a nivel mundial; en Colombia es un cultivo con gran potencial de exportación. Sin embargo, insectos plaga como los trips, pueden incidir en su calidad y producción. En esta investigación se evaluó la relación de la fluctuación poblacional de trips (Frankliniella cf. gardeniae) con las condiciones climáticas, la fenología del mango (var. Yulima y Tommy) y los hospederos alternos en el Guamo y San Luis (Tolima). Semanalmente se muestrearon trips en inflorescencias, hojas jóvenes de mango y en arvenses asociadas, se registraron los estados fenológicos del cultivo, las condiciones climáticas (temperatura, humedad relativa y precipitación) y se determinó la probabilidad de presencia de trips en inflorescencias de mango. En total se recolectaron 8.609 individuos de trips entre adultos y ninfas. La fluctuación poblacional de $F$. cf. gardeniae presentó relación positiva con la floración $(\mathrm{r}=0,79$ var. Yulima y Tommy) y la temperatura $(\mathrm{r}=0,43$ var. Yulima; $\mathrm{r}=0,48$ var. Tommy). Se registraron los géneros fitófagos Frankliniella y Haplothrips, en las arvenses Desmodium tortuosum y Melochia parvifolia. Las flores y frutos hasta tamaño de $8 \mathrm{~mm}$ fueron las más susceptibles al ataque del insecto. Finalmente, la mayor probabilidad de presencia de trips en inflorescencias de mango presentó dos períodos marcados, de 7 a 63 y de 161 a 203 días después de floración. Frankliniella cf. gardeniae en mango se comportó como una plaga ocasional, presente cuando la fuente de alimento estuvo disponible y las condiciones climáticas fueron adecuadas.
\end{abstract}

Palabras clave: Fenología, inflorescencia, floración, plaga, condiciones climáticas.

\begin{abstract}
Mango is one of the tropical fruits with the highest production worldwide; in Colombia is a crop with a great export potential. However, insect pests such as thrips, can affect its quality and production. In this research the relationship of the population fluctuation of thrips (Frankliniella $\mathrm{cf}$. gardeniae) with climatic conditions and mango (var. Yulima and Tommy) phenology in Guamo and San Luis (Tolima) was evaluated. Trips were weekly sampled in both inflorescences and young leaves of mango and associated weeds; phenological stages of the crop and climatic conditions (temperature, relative humidity and precipitation) were recorded and the probability of the presence of thrips in mango flowers was determined. A total number of 8,609 thrips individuals, including adults and immatures, were collected. The population fluctuation of Frankliniella $\mathrm{cf}$. gardeniae presented a positive relationship with flowering $(\mathrm{R}=$ 0.79 var. Yulima and Tommy) and temperature $(R=0.43$ var. Yulima; $R=0.48$ var. Tommy). It was recorded the genera of the phytophagous trips Frankliniella and Haplothrips, in the weeds Desmodium tortuosum and Melochia parvifolia. The flowers and fruits up to $8 \mathrm{~mm}$ size were the most susceptible to insect attack. Finally, the higher probability of the thrips presence in mango flowers had two main periods, 7-63 days and 161-163 days after flowering. Frankliniella cf. gardeniae behaved as an occasional pest, present when the food source was available and the climatic conditions were adequate.
\end{abstract}

Key words: Phenology, inflorescence, flowering, pest, climatic conditions.

\section{Introducción}

El cultivo de mango (Mangifera indica L., 1753) ha sido de gran importancia a nivel mundial, debido a que es una de las frutas tropicales con mayor producción (FAO 2012). En Colombia, el Tolima es uno de los principales productores con el 26,6 \% del área total (SIOC 2018); sin embargo, en 2017, este departamento presentó un área de siembra con una tendencia a la baja reportando 7.028 ha, en comparación con 7.328 ha en 2016 (Sistema de información de gestión y desempeño de organizaciones de cadenas, SIOC 2018). Estos cambios a nivel de áreas de producción, generalmente se asocian entre otras cosas a prácticas agrícolas inadecuadas y a la aparición de problemas fitosanitarios, como insectos plaga, dentro de los que se pueden incluir las especies de trips (Gil et al. 2013), las cuales se alimentan de hojas, flores y frutos en crecimiento, lo que genera manchas, cicatrices y caída prematura, afectando la calidad y producción (Aguirre et al. 2013).

Los trips (Thysanoptera) comprenden unas 6.000 especies, en este orden se encuentran dos subórdenes de importancia, Tubulifera y Terebrantia (Soto-Rodríguez et al. 2012). Se estima que alrededor de 100 especies pueden ser plagas, debido a que se alimentan de diferentes tejidos vegetales (Monteiro et al. 2001). Los trips son macroinvertebrados comunes en el trópico y subtrópico, pero a pesar de la gran relevancia en estas regiones se tiene poco conocimiento sobre su abundancia estacional y las condiciones que favorecen su

\footnotetext{
${ }^{1}$ Ingeniero Agrónomo, Corporación Colombiana de Investigación Agropecuaria-Agrosavia, C.I. Nataima Km 9 vía Espinal-Chicoral, Tolima, Colombia, psierra@agrosavia.co. ${ }^{2}$ Ph. D. Corporación Colombiana de Investigación Agropecuaria-Agrosavia, C.I. Nataima Km 9 vía Espinal-Chicoral, Tolima, Colombia, evaron@agrosavia.co. Código Orcid: https://orcid.org/0000-0002-5421-507X. ${ }^{3} \mathrm{Ph}$. D. Docente de planta Universidad de Caldas. Cl. 65 \#2610 Manizales, Caldas, lucimardias@ucaldas.edu.co. ${ }^{4}$ Ingeniero Agrónomo, Corporación Colombiana de Investigación Agropecuaria-Agrosavia, C.I. Nataima Km 9 vía Espinal-Chicoral, Tolima, Colombia, cijaramillo@agrosavia.co. Código Orcid: https://orcid.org/0000-0002-8302-2736. Autor para correspondencia: Edgar Herney Varón-Devia. Corporación Colombiana de Investigación Agropecuaria-Agrosavia, C.I. Nataima Km 9 vía Espinal-Chicoral, Tolima, Colombia,evaron@agrosavia.co.
} 
población en cultivos como el mango. Las condiciones ambientales como la temperatura, humedad y precipitación pueden influir en la densidad de estas plagas (Leite et al. 2006; Wang y Tong 2012). La distribución, abundancia y comportamiento de estos insectos, es muy afectada por la temperatura, por lo que conocer y registrar las condiciones climáticas de la región, debido a que pueden brindar una herramienta para formular planes de control de los insectos plagas (Yadav y Chang 2014).

Las poblaciones de trips no solo dependen de los factores Las poblaciones de trips no solo dependen de los factores climáticos, sino también del alimento disponible en el cultivo; en uva, por ejemplo, poblaciones de Frankliniella occidentalis (Pergande, 1895) se incrementan en el estado de floración, debido a que los adultos se alimentan del polen (Lopes et al. 2002). F. gardeniae (Moulton, 1948) (Thripidae) es una especie fitófaga que tiene preferencia por los órganos florales, brotes tiernos y frutos en desarrollo de aguacate (Duarte de Oliveira et al. 2011) entre otros frutales. En mango, esta especie se alimenta de las inflorescencias y cuando éstas entran en senescencia las poblaciones disminuyen, lo anterior evidencia una sincronía entre las poblaciones de trips y la floración (Retana-Salazar y Rodríguez-Arrieta 2015). Malasia, China, México y Brasil reportan la mayor información de la plaga, siendo $F$. gardeniae la especie más común en Latinoamérica (México y Brasil) debido a que presenta una alta incidencia en panículas de inflorescencias de mango (Aliakbarpour y Rawi 2012; Duarte de Oliveira et al. 2011; García 2012; Rocha et al. 2012). En Colombia los estudios relacionados de trips en mango son muy escasos. Sierra (2017), realizó un estudio en cultivos de mango, el cual se basó en determinar dos parámetros importantes de la plaga como son el nivel de daño económico y la fluctuación poblacional del insecto en el cultivo. El presente artículo se deriva de ese estudio, y contribuye a generar criterios para un manejo integrado y económico de la plaga, que permitan aumentar la productividad del mango. Por lo anterior, esta investigación evaluó la fluctuación poblacional de trips (Frankliniella $\mathrm{cf}$ gardeniae) con relación a la fenología del cultivo (floración, vegetativo, fructificación y reposo) y a las variables climáticas (temperatura, humedad relativa y precipitación) en mango (Mangifera indica L., var. Yulima y Tommy) en los municipios del Guamo y San Luis (Tolima) y determinar su presencia en arvenses aledañas al cultivo.

\section{Materiales y métodos}

Ubicación geográfica. El estudio se realizó en dos fincas de mango de los municipios del Guamo (4'01'41" N y $74^{\circ} 58^{\prime}$ 12 " O y $326 \mathrm{msnm}$ de altitud) y San Luis $\left(4^{\circ} 07^{\prime} 56^{\prime}\right.$ " N y $75^{\circ}$ 05'44" O, altitud de $500 \mathrm{msnm}$ ), departamento del Tolima. Los cultivos de mango (var. Yulima y Tommy) presentes en las dos fincas constaban de árboles con alturas entre 5 y $8 \mathrm{~m}$, una edad entre los 9 a 15 años, densidad de siembra de 156 árboles $\mathrm{ha}^{-1}$ con un área total de 60 ha aproximadamente para cada finca.

Fluctuación poblacional en campo y fase de laboratorio. Se registraron datos durante los semestres B de 2015 y A de 2016, para un total de 44 semanas de muestreo. La unidad experimental fue una parcela de 16 árboles por cada variedad (Yulima y Tommy), en la que se evaluaron al azar cuatro árboles por muestreo. Se seleccionó aleatoriamente una hoja (terminal y joven) del tercio bajo y una inflorescencia (abierta y frutos recién formados) por árbol, como unidad de muestreo. Los terminales muestreados presentaban hojas que no tenían la separación ni alongación de su tamaño final; las hojas jóvenes se caracterizaron por tener un color verde brillante y tener un $30 \%$ del tamaño definitivo (García 2011). Las inflorescencias consideradas óptimas para el muestreo fueron aquellas alongadas y con flores abiertas, también se evaluaron inflorescencias fecundadas (algunas con flores abiertas pero la mayoría tenía frutos en desarrollo con diámetros promedio de 2,55 $\pm 0,28 \mathrm{~cm}$ ) (García 2011).

El método de muestreo que se implementó fue por aspersión directa a las estructuras vegetales, mediante un recipiente plástico con atomizador que contenía una solución de

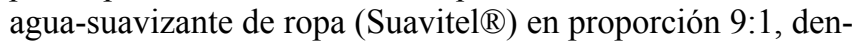
tro una bolsa de plástico con cierre hermético (Ziploc $®$ de 27 x $28 \mathrm{~cm}$ ) que se ubicó debajo de la estructura (Ascención-Betanzos et al. 1999). El muestreo se realizó una vez por semana. Se hicieron los conteos y la separación por morfoespecies de los trips con ayuda de un estereoscopio (Leica, EZ4), se almacenaron en alcohol al 70 \% en el laboratorio de Entomología del centro de investigación Nataima de Agrosavia hasta su identificación taxonómica por Everth Ebratt Ravelo (M. Sc.), en el laboratorio de diagnóstico fitosanitario del ICA, Bogotá y Élison Fabricio Bezerra Lima (taxónomo especialista UFPI/CAFS, Brasil). Se estimó la densidad poblacional de trips al promediar el número de individuos presentes en las inflorescencias y/o hojas muestreadas. Los estados fenológicos (vegetativo, reposo, floración y fructificación) del cultivo se registraron semanalmente en porcentaje (\%) por cada árbol muestreado. Los datos climáticos [temperatura promedio $\left({ }^{\circ} \mathrm{C}\right)$, precipitación acumulada $(\mathrm{mm})$ y humedad relativa promedio (\%)] se tomaron con estaciones meteorológicas (Davis, Weather Link 6.0), en las fincas objeto del estudio.

La presencia de trips en arvenses asociadas al cultivo de mango en las fincas objeto de estudio fue registrada semanalmente. Las arvenses se muestrearon con el método del golpeteo de la inflorescencia (Avellaneda et al. 2016), que consistió en golpear la arvense tres veces sobre una malla metálica puesta sobre un plato de aproximadamente $5 \mathrm{~cm}$ de profundidad (con el fin de facilitar el conteo de trips). Las arvenses muestreadas estaban a tres distancias del árbol de mango (0-3, 3-6 y 6-9) $\mathrm{m}$ aprox. La unidad de muestreo seleccionada fue una arvense (en estado de floración) por cada distancia del árbol evaluada. El muestreo se realizó en horas de la mañana y se evaluó por 44 semanas con 163 muestras de arvenses en total; las arvenses que se registraron con mayor frecuencia en los árboles de mango tuvieron un total de diez repeticiones en el tiempo. Las muestras de trips se llevaron para identificación taxonómica al laboratorio de diagnóstico fitosanitario del ICA, Bogotá. Una muestra de los especímenes de trips reposa en el área de trabajo de entomología de Agrosavia CI Nataima (El Espinal, Tolima) y en la Colección Taxonómica Nacional de Insectos "Luis María Murillo" del CI Tibaitatá (Mosquera, Cundinamarca) con número de catá$\log 2515$.

Probabilidad de presencia de trips en el cultivo de mango. Se determinó la probabilidad de presencia de trips en el cultivo de mango durante las semanas de muestreo, las cuales incluyeron dos períodos de floración (semestres B 2015 y A de 2016). Para esto se transformó la variable de número de trips a presencia (1) o ausencia (0), y después se realizó un modelo 
de regresión logística longitudinal con la siguiente función:

$$
\pi_{\mathrm{i}}=\begin{gathered}
\exp \left(\beta 0+\beta 1 \text { Variedad }_{\mathrm{i}}+\beta 2 D D F+\beta 3 \text { Localidad }\right) \\
1+\exp \left(\beta 0+\beta 1 \text { Variedadi }^{2}+\beta 2 D D F+\beta 3 \text { Localidad }\right)
\end{gathered}
$$

Dónde: $\pi_{\mathrm{i}}$ es la probabilidad de que se encuentre un trips en un muestreo i. $\beta 0$ : intercepto. $\beta 1$ : Coeficiente de la variable variedad. $\beta 2$ : Coeficiente de la variable días después de floración (DDF), contados a partir del inicio del primero de los dos períodos de floración que incluyó el estudio. $\beta 3$ : Coeficiente de la variable localidad (municipio). La significancia de cada variable se comparó por medio de la prueba de Wald.

Análisis estadístico. Se realizaron comparaciones del promedio de trips por inflorescencia de mango entre municipios, variedades y periodos de floración evaluados, mediante la prueba no paramétrica de Wilcoxon-Mann-Whitney, debido a que los datos no presentaron una distribución normal (ShapiroWilk $\mathrm{P}=<0,0001)$. Por otro lado, se hicieron correlaciones mediante el coeficiente de correlación de Spearman entre el número de trips por órgano por semana y los datos climáticos y fenológicos del cultivo con el software estadístico SAS (SAS Institute 2009). Los análisis estadísticos de probabilidad de presencia de trips en inflorescencias se hicieron mediante el software estadístico R (R Project 2016).

\section{Resultados}

Se encontraron poblaciones muy bajas de trips en terminales y hojas jóvenes (var. Tommy $=0,28 \pm 0,11$; var. Yulima $=$ $0,12 \pm 0,03$ trips por semana por hoja), por lo que no fueron representativas, razón por la cual se realizaron los análisis a las poblaciones de trips encontradas en inflorescencias (abiertas) y frutos recién formados, donde fueron altas.

No se encontraron diferencias estadísticamente significativas para los promedios de trips por inflorescencia de mango entre municipios $(\operatorname{Pr}<\mathrm{Z}=0,1938)$, ni variedades $(\operatorname{Pr}<\mathrm{z}=$ $0,6125)$; siendo las medias más altas las que se registraron en San Luis, tanto en la variedad Tommy con 39,57 \pm 9,21, como Yulima con 16,56 $\pm 4,41$. En el municipio del Guamo se presentaron medias más bajas en las dos variedades, Yulima con $9,35 \pm 1,89$ y Tommy con $8,62 \pm 2,83$.

Por su parte, se compararon los dos periodos de floración por separado y fueron las medias del semestre B de 2015 (semanas 1-9 de muestreo) las que presentaron los mayores valores de número de trips en inflorescencias de mango, pero sin diferencias estadísticamente significativas $(\operatorname{Pr}<\mathrm{Z}=$ 0,7463 ) con las medias del semestre A de 2016 (semanas del 23-29 de muestreo) (Tabla 1).

Relación entre la fluctuación poblacional y la fenología. En la variedad Yulima la fluctuación poblacional de trips se relacionó directamente con la floración: $\mathrm{r}=0,7674 ; \mathrm{P}=<$ 0,0001 para el municipio del Guamo (Fig. 1A) y r $=0,7998$ (P $=<0,0001)$ para San Luis (Fig. 1B). La fluctuación de trips presentó una relación negativa con el estado fenológico de reposo $(\mathrm{r}=-0,6335 ; \mathrm{P}=<0,0001$ para el Guamo y $\mathrm{r}=-0,3858$; $\mathrm{P}=0,0033$ para San Luis) y fructificación en San Luis $(\mathrm{r}=$ $-0,3661 ; P=0,0145$ ); el estado vegetativo no presentó relación significativa con la fluctuación de trips en Yulima $(\mathrm{r}=$ 0,$2257 ; \mathrm{P}=0,1406$ para el Guamo y $\mathrm{r}=0,2593 ; \mathrm{P}=0,0891$ para San Luis).

En la variedad Tommy la fluctuación del insecto presentó una relación directa con la floración en el Guamo ( $\mathrm{r}$ $=0,7951 ; \mathrm{P}=<0,0001$; Fig. $1 \mathrm{C})$ y San Luis $(\mathrm{r}=0,7528$ $(\mathrm{P}=<0,0001$; Fig. 1D). El estado de reposo en esta variedad presentó una relación inversamente proporcional con la fluctuación de trips en los dos municipios (Guamo $\mathrm{r}=$ $-0,5307 ; \mathrm{P}=0,0002$ y San Luis $\mathrm{r}=-0,3500 ; \mathrm{P}=0,0198$ ). La fructificación no registró una relación significativa con la fluctuación de trips en ninguno de los dos municipios evaluados (Guamo con un $\mathrm{r}=0,1768 ; \mathrm{P}=0,2508$ y San Luis con un $\mathrm{r}=0,1253 ; \mathrm{P}=0,4175)$.

Relación entre la fluctuación poblacional y las variables climáticas. En el Guamo la temperatura presentó una relación directa con las poblaciones de trips en las dos variedades evaluadas. Para Yulima se registró un coeficiente de $r=0,3993$ $(\mathrm{P}=0,0073)$ (Fig. 2A) y en Tommy se obtuvo un $\mathrm{r}=0,4816$ $(\mathrm{P}=0,0009$; Fig. 2B). Por el contrario, la humedad relativa tuvo una influencia negativa en la fluctuación de trips en las dos variedades (Yulima: $\mathrm{r}=-0,4559 ; \mathrm{P}=0,0019$ y Tommy: $\mathrm{r}$ $=-0,6075 ; \mathrm{P}=0,0001)$. Para la precipitación acumulada, no se evidenció una relación estadísticamente significativa con la fluctuación de trips en ninguna de las variedades evaluadas ( $\mathrm{P}=0,3921$ para Yulima y $\mathrm{P}=0,4156$ para Tommy).

En San Luis se registró una relación negativa de la fluctuación de trips con la humedad relativa en las dos variedades

Tabla 1. Promedios \pm EE de trips por inflorescencia de mango en las variedades (Yulima y Tommy) y en los municipios evaluados (Guamo y San Luis) durante los periodos de floración de los semestres B de 2015 y A de 2016.

\begin{tabular}{lccc}
\hline Municipio & Variedad & $\begin{array}{c}\text { Periodo de floración } \\
\text { del mango }\end{array}$ & $\begin{array}{c}\text { Promedio de trips por } \\
\text { inflorescencia }\end{array}$ \\
\hline \multirow{2}{*}{ Guamo } & Tommy & B-2015 & $29,19 \pm 10,99^{\mathrm{a}^{*}}$ \\
& \multirow{2}{*}{ Yulima } & A-2016 & $11,24 \pm 3,35^{\text {a }}$ \\
\cline { 2 - 4 } & \multirow{2}{*}{ Tommy } & B-2015 & $17,71 \pm 4,85^{\text {a }}$ \\
& & A-2016 & $18,87 \pm 4,57^{\text {a }}$ \\
\hline \multirow{2}{*}{ San Luis } & \multirow{2}{*}{ Yulima } & B-2015 & $92,76 \pm 21,43^{\text {a }}$ \\
& & A-2016 & $51,31 \pm 15,84^{\text {a }}$ \\
\cline { 2 - 4 } & & B-2015 & $31,98 \pm 9,51^{\text {a }}$ \\
& & A-2016 & $57,01 \pm 14,59^{\text {a }}$ \\
\hline
\end{tabular}

*Letras iguales indican que no hubo diferencia estadísticamente significativa. 

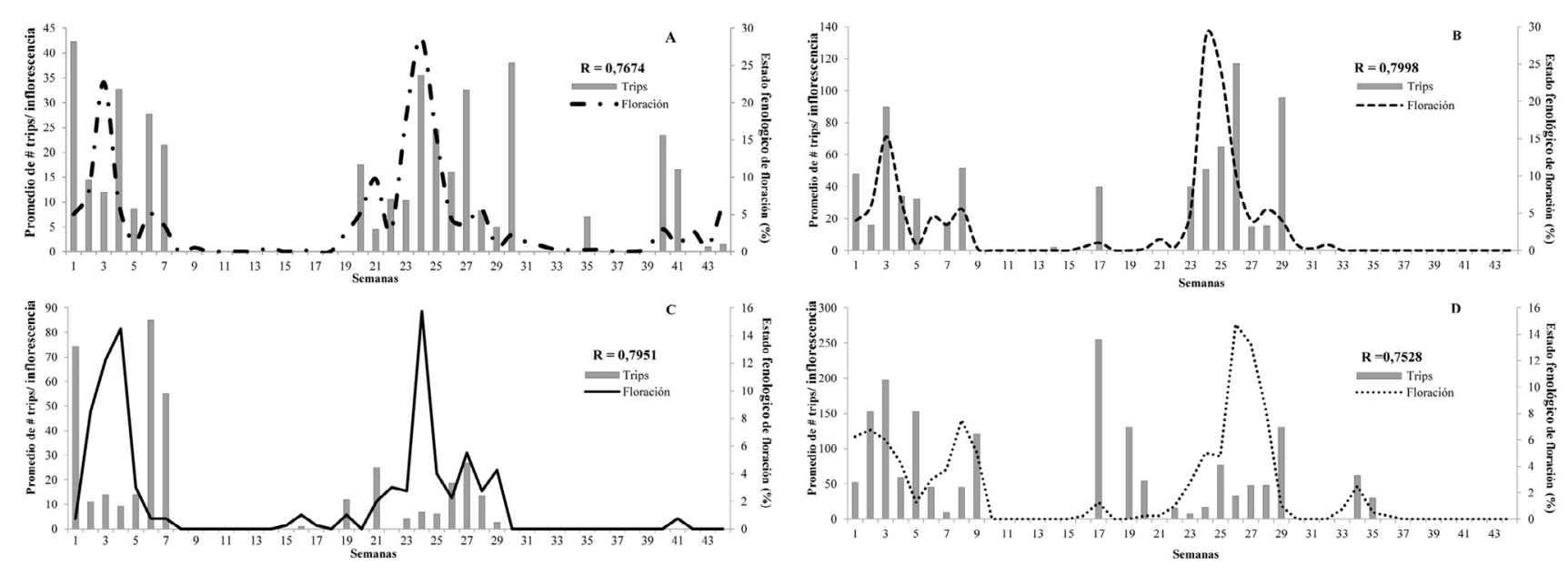

Figura 1. Fluctuación poblacional del número de trips (Frankliniella. cf. gardeniae) en inflorescencias de mango (Mangifera indica) con relación al estado fenológico de floracion (\%), B-2015 y A-2016. A. Var. Yulima, municipio del Guamo. B. Var. Yulima, municipio de San Luis. C. Var. Tommy, municipio del Guamo. D. Var. Tommy, municipio de San Luis.

(Yulima: $\mathrm{r}=-0,3528 \mathrm{P}=0,0188$ y Tommy: $\mathrm{r}=-0,4273 \mathrm{P}$ $=0,0038)$, a diferencia de la temperatura, la cual tuvo una influencia positiva (Yulima: $r=0,4306 \mathrm{P}=0,035$; Fig. $2 \mathrm{C}$ y Tommy: $\mathrm{r}=0,4771 \mathrm{P}=0,0011$; Fig. 2D). La precipitación acumulada no presentó relación estadísticamente significativa con la fluctuación de la plaga (Yulima: $\mathrm{P}=0,8961 \mathrm{y}$ Tommy: $\mathrm{P}=0,5452$ ).

Trips en arvenses asociadas al mango. Los trips que se registraron pertenecen a los géneros fitófagos Frankliniella y Haplothrips. En San Luis Desmodium tortuosum (Sw. DC 1825) (Fabales: Leguminosae), fue la arvense que registró mayor presencia de trips en las dos variedades de mango $(92,77 \%$ para Tommy y $81,82 \%$ para Yulima). Por su parte, Melochia parvifolia (Kunth 1823) (Malvales: Sterculiaceae) fue la que presentó mayor más abundancia en las dos variedades evaluadas (62,50\% para Tommy y 43,60 \% para Yulima) en Guamo.

Los trips se registraron en mayor abundancia en las arvenses muestreadas a una distancia de 3-6 $\mathrm{m}$ del árbol de mango (zona de influencia del surco), en los dos municipios y variedades evaluadas (var. Yulima 159 y 104 individuos y
var.Tommy 56 y 75 individuos en el Guamo y San Luis respectivamente). En abundancia de trips, la segunda mayor se presentó en la distancia de 0-3 m del árbol (zona de influencia de la copa) en la variedad Yulima, en la que se registraron 12 y 63 individuos en arvenses asociadas al cultivo en Guamo y San Luis, respectivamente. En la variedad Tommy, la segunda mayor abundancia de trips se presentó a una distancia de 6-9 m del árbol de mango (zona del borde del surco cerca al árbol muestreado), con 46 individuos en Guamo y 62 en San Luis.

Probabilidad de presencia de trips en el cultivo de mango. Se presentó diferencia estadísticamente significativa entre los $\mathrm{DDF}(\mathrm{P}=0,0001)$ para la probabilidad de presencia de trips en el cultivo. Por el contrario, no hubo significancia estadística en la variable municipio $(\mathrm{P}=0,7246)$, con promedios de probabilidad de presencia de trips de $25,37 \% \pm 3,16$ para el Guamo y $26,44 \% \pm 3,15$ para San Luis. Tampoco se presentó diferencia estadística en la variedad $(\mathrm{P}=0,5271)$ con promedios de 26,52 $\% \pm 3,21$ para Tommy, y Yulima con $25,09 \% \pm 3,12$.

La probabilidad de presencia de trips en inflorescencias de mango presentó dos periodos marcados, el primero de los
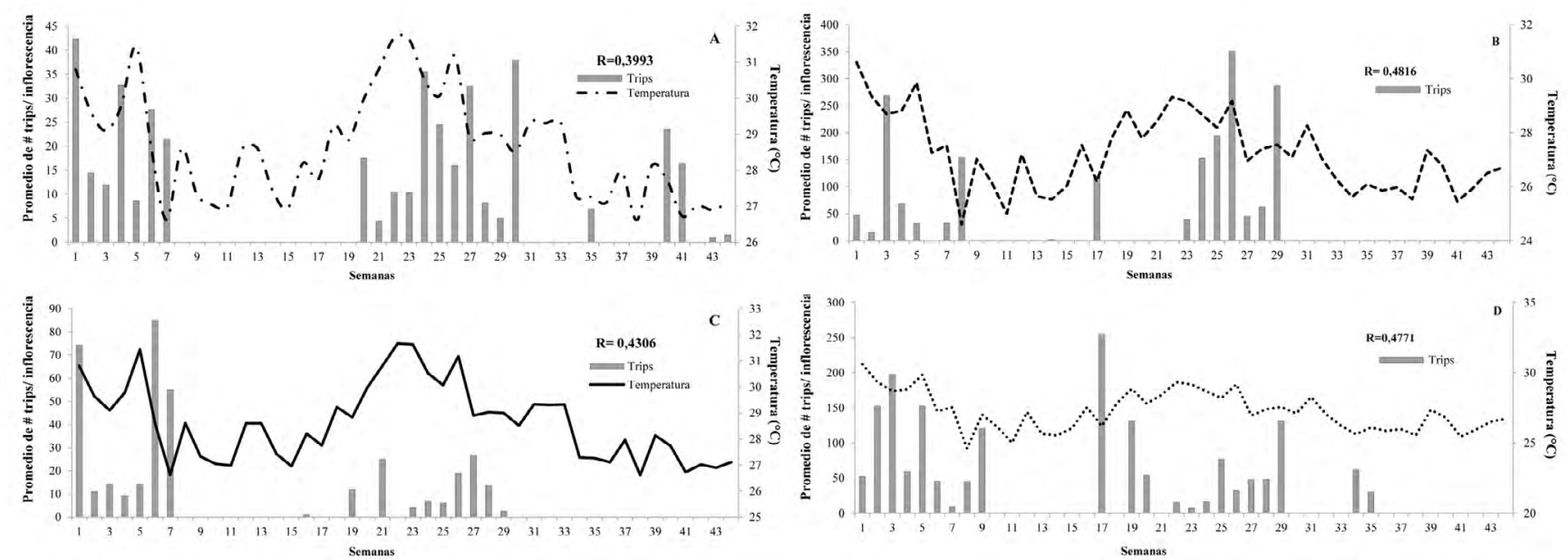

Figura 2. Fluctuación poblacional del número de trips (Frankliniella. cf. gardeniae) en inflorescencias de mango (Mangifera indica) con relación a la variable climática de temperatura promedio $\left({ }^{\circ} \mathrm{C}\right)$ B-2015 y A-2016. A. Var. Yulima, municipio del Guamo. B. Var. Tommy, municipio del Guamo. C. Var. Yulima, municipio del San Luis. D. Var. Tommy, municipio de San Luis. 


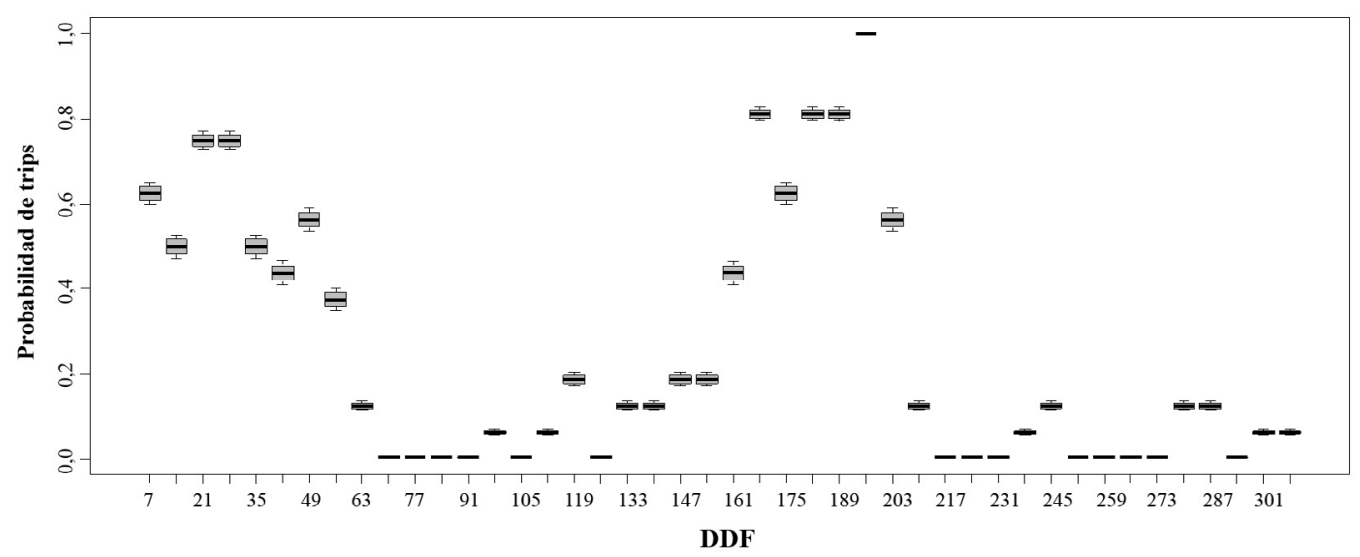

Figura 3. Probabilidad de presencia de trips (Frankliniella. cf. gardeniae) en inflorescencias de mango durante 308 días después de inicio de floración (DDF) en los semestres B-2015 y A-2016.

7 a 63 días despues de inicio de floración (DDF) y el segundo de los 161 a 203 DDF; siendo el segundo el que presentó mayor probabilidad de presencia del insecto alcanzando valores del $100 \%$, por el contrario en los días intermedios y posteriores a los dos periodos, los valores fueron muy bajos con 2,84 $\%$ (Fig. 3).

Por su parte, la probabilidad de presencia de trips en inflorescencias fueron muy similares, con máximos en el primer periodo de 77,04 \% en San Luis y 75,54 \% en el Guamo a los 21 DDF en la variedad Tommy y máximos de 74,50 $\%$ para San Luis y $72,90 \%$ para el Guamo a los 21 DDF en Yulima.

El segundo periodo alcanzó $100 \%$ de probabilidad de presencia de trips a los 196 DDF en las dos variedades y los dos municipios. A los 308 DDF (44 semanas de muestreo) se registró la menor probabilidad de presencia de trips con 2,84 $\%$ en la variedad Yulima y 3,09 \% para Tommy en el municipio del Guamo. En San Luis los valores mínimos de probabilidad de presencia de trips en inflorescencias fueron similares con 3,09\% para Yulima y 3,54\% para Tommy. Los periodos de mayor probabilidad en las dos variedades y municipios coincidieron con las dos etapas de floración de mango de los semestres del estudio, y las menores probabilidades fueron registradas cuando el cultivo estaba en etapas de reposo y fructificación.

\section{Discusión}

En el presente estudio el estado de floración de los árboles de mango contribuyó al aumento poblacional de trips; lo que concuerda con las investigaciones de Durán (2012) y García (2012) en México quienes encontraron que las altas poblaciones del insecto se presentaron principalmente en floración y frutos con tamaños de $8 \mathrm{~mm}$ de diametro, indicando que la presencia de esta plaga se favoreció con el estado fenológico de floración.

Por su parte, la población de trips en los brotes vegetativos y hojas jóvenes fue muy baja, concordando con lo citado por García (2012) quien expresó que estos órganos al no ser alimento óptimo para los trips, ocasionan que se presenten en bajas poblaciones y que no sean una amenaza para el área foliar del cultivo de mango. Diferentes autores reportaron que el daño causado en las inflorescencias de mango de los cultivos de México y Brasil fue principalmente ocasionado por los trips F. invasor (Sakimura 1972) y F. gardeniae (Duarte de Oliveira et al. 2011; Durán 2012; García 2012; Rocha et al. 2012; Rocha et al. 2015; Salazar 2012). El daño de un insecto puede variar según la especie y el cultivo atacado. En aguacate por ejemplo Urías-López et al. (2007), reportaron el ataque de trips tanto en floración como en etapa vegetativa del cultivo; en mango Scirtothrips dorsalis (Hodd 1919) (Thripidae) registró poblaciones que migran a las hojas tiernas una vez ha pasado la floración, lo que genera la caída de las flores (Chiu et al. 2012; De la Rosa 2010; Munj et al. 2012).

En este estudio, la población de trips (Frankliniella $\mathrm{cf}$. gardeniae) se redujo cuando los árboles de mango estaban en estado de reposo o fructificación, debido a que disminuyó la cantidad de alimento óptimo y el insecto se refugió en plantas huéspedes (Aliakbarpour y Rawi 2012; De la Rosa 2010). Conocer los hábitos alimenticios de la especie que esté causando daño en el cultivo de interés, permite establecer un plan de manejo eficiente para la plaga (Capetillo 2013). Debido a que algunas especies de trips en mango $[F$. cephalica (Crawford 1910) (Thripidae) y F. gardeniae] son antófilas (Retana-Salazar y Rodríguez-Arrieta 2015), la población disminuye naturalmente cuando ha pasado la época de floración en el cultivo, por tanto, debe centrarse su control en esta etapa fenológica. Estos insectos antófilos procesan más fácil los alimentos simples como aminoácidos libres y azúcares, los cuales se concentran en la floración y brotación de hojas de las plantas, siendo las épocas más susceptibles al ataque (Chaboussou 1987 citado por Santos 2010). No sólo se alimentan de estos órganos, sino que en algunos casos también muestran preferencia de oviposición como fue reportado por Ortiz et al. (2016), donde $F$. invasor prefirió ovipositar en los botones florales y flores de mango con una duración de huevo a adulto de 189,8 horas (7,9 días).

En un estudio en mango, realizado en Taiwan, ChunNan et al. (2015) ejecutaron un modelo de regresión logística, en el cual determinaron los factores que más influyeron en la población de trips ( $S$. dorsalis), siendo la época de floración y los cultivares de mango (variedades Irwin, JinHuang y Tsar-Swain) los que favorecieron la presencia del insecto, a diferencia de lo presentado en este estudio en el cual las variedades evaluadas no influyeron en la población de trips.

La probabilidad de presencia de trips en inflorescencias a partir del modelo logístico ejecutado, mostró diferencia 
en los días después de inicio de floración, lo que permitió identificar dos periodos (7 a 63 y 161 a 203 DDF) con altas probabilidades (hasta $100 \%$ ) de encontrar la plaga en inflorescencias. Estos dos periodos de altas probabilidades coincidieron con los de floración de los semestres estudiados, dejando en evidencia la estrecha relación de los trips con la etapa de floración del mango. Resultados similares arrojaron las correlaciones de la fluctuación de la plaga con los estados fenológicos del mango, en el que se presentó una relación positiva entre la densidad de trips y la floración del mango, en las dos variedades y en los dos municipios objeto de estudio. Por el contrario, los estados fenológicos de reposo y fructificación, que no favorecieron la fluctuación poblacional de trips en el mango, debido a que al estar los árboles en estas etapas fenológicas se disminuye la fuente de alimento para el insecto.

No solo los estados fenológicos del cultivo influyen en la fluctuación de un insecto, tambien existen otros factores como los climáticos que pueden contribuir en el aumento o disminución poblacional de una plaga. Por ejemplo, en cultivos de mango de Malasia se reportó el incremento de los trips cuando se presentaron epocas secas y floración de los árboles, y tambien se registró una disminución de la plaga en presencia de lluvias y bajas temperaturas (Aliakbarpour y Rawi 2012). La temperatura en temporadas secas es mayor, y para el caso la población de $S$. dorsalis fue la condicion climática más influyente, debido a que aportó un 23,39 del $80 \%$ de los factores evaluados en el modelo de regresión logistica por Chun-Nan et al. (2015); otras condiciones que incidieron pero negativamente fueron la humedad relativa $(22 \%)$ y la precipitación $(8,33 \%)$. Otros autores que concuerdan con la influencia de las condiciones climáticas en la poblacion de trips [Scirtothrip citri (Moulton, 1909) (Thripidae)] son López-Guillén et al. (2014); quienes reportaron altas poblaciones del insecto cuando la humedad relativa fue baja $(70-80 \%)$ y la precipitación escasa $(0,15$ $6,13 \mathrm{~mm}$ ).

Debido a que los trips en mango son plagas ocasionales porque su población decrece cuando el alimento óptimo en el cultivo se agota (Durán 2012; García- Escamilla et al. 2016), su control no solamente debe estar dirigido a la etapa fenológica de floración, sino también a las arvenses hospederas cercanas que pueden servir como refugio de los trips cuando el mango no tiene inflorescencias (Aliakbarpour y Rawi 2012; De la Rosa 2010), por tanto, es aconsejable llevar a cabo un manejo del insecto durante la floración de las arvenses hospederas con el fin que no sirvan de alimento o refugio para la plaga, el cual puede consistir en el monitoreo de poblaciones y la eliminación de arvenses en floración en áreas con altas densidades poblacionales de trips (Solís 2016).

Las arvenses registradas como hospederas de trips en mango en Malasia fueron Mimosa púdica L., Cleome rutidosperma DC., Echinochloa colonum (L.) Link 1833, Borreria laevicaulis (Miq.) Ridl., Veronia cinerea (L.) Less. y Asystasia coromandeliana (L.) T. Anderson (Aliakbarpour y Rawi 2012), en los resultados obtenidos en este estudio se registró mayor presencia de trips en las arvenses $D$. tortuosum y M. parvifolia. Por su parte, los géneros de trips fitofagos Frankliniella y Haplothrips encontrados en arvenses asociadas al cultivo del mango en este estudio, también fueron reportados en inflorescencias de mango en México y Malasia (Aliakbarpour y Rawi 2012; Rocha et al. 2012).
En síntesis, los hallazgos registrados en este estudio coinciden con lo citado por diferentes autores, debido a que la población de trips en las dos variedades (Tommy y Yulima) y municipios (Guamo y San Luis) evaluados, presentó un incremento a mayor temperatura y decreció cuando la humedad relativa fue alta. A partir del monitoreo de las condiciones ambientales sumado a un muestreo oportuno de la poblacion de trips en la época más susceptible del cultivo de mango (floración), se podría implementar un plan de manejo integral que incluya el control de arvenses, con el que se logre controlar la plaga de manera eficiente.

\section{Conclusiones}

En cultivos de mango (var. Tommy y Yulima) de los municipios de San Luis y Guamo (Tolima), la población de trips (F. cf. gardeniae) actuó como una plaga ocasional, estando presente en el cultivo en el estado de floración. La población del insecto se vio favorecida por las altas temperaturas y por la presencia de las arvenses asociadas al cultivo Desmodium tortuosum y Melochia parvifolia. Para el manejo del insecto en el cultivo de mango se deben tener en cuenta la fenología, las condiciones climáticas y las arvenses asociadas presentes.

\section{Agradecimientos}

Agradecemos a la Corporación Colombiana de Investigación Agropecuaria "Agrosavia" y al Ministerio de Agricultura y Desarrollo Rural "MADR", por el apoyo en la ejecución de esta investigación con tiempo y recursos financieros. Al Departamento del Tolima, a la Universidad del Tolima, a la Universidad de Ibagué, Ceiba y proyecto "Formación de Talento Humano de Alto Nivel"; por la inmensa ayuda brindada y a la Universidad de Caldas por los conocimientos adquiridos durante mi maestría en el desarrollo de esta investigación. A los doctores Élison Fabricio Bezerra Lima (UFPI/CAFS, Brasil) y Ever Ebratt, por su apoyo en las identificaciones taxonómicas.

\section{Literatura citada}

AGUIRRE, A.; MIRANDA, M.; URÍAS, M.; ORONA, F.; ALMEYDA, I.; JOHANSEN, R.; TUCUCH, M. 2013. Especies de trips (Thysanoptera) en mango, fluctuación y abundancia. Revista Colombiana de Entomología 39 (1): 9-12.

ALIAKBARPOUR, H.; RAWI, C. 2012. The species composition of thrips (Insecta: Thysanoptera) inhabiting mango orchards in Pulau Pinang, Malaysia. Disponible en: http://www.pubfacts. com/detail/24575225/The-species-composition-of-thrips-insecta-thysanoptera-inhabiting-mango-orchards-in-pulau-pinangmal. [Fecha revisión: 12 febrero 2016].

ASCENSIÓN-BETANZOS, G.; BRAVO-MOJICA, H.; GONZÁLEZ-HERNÁNDEZ, H.; JOHANSEN-NAIME, R.; BECERRIL-ROMÁN, A. 1999. Fluctuación poblacional y daño de trips en aguacate CV. Hass. Revista Chapingo 5: 291-296.

AVELLANEDA N., J. A.; DIAZ T., M. A., ACOSTA LEAL, D. A.; RODRIGUEZ C., D.; CAPETILLO, E. 2013. Fluctuación poblacional del trips de banda roja, Selenothrips rubrocinctus (Giard) (Thysanoptera: Thripidae) en el cultivo de cacao, en la Chontalpa Tabasco, México. Tesis de maestría en Ciencias, Colegio de postgrados, Institución de enseñanza e investigación en ciencias agrícolas. Montecillo, $47 \mathrm{p}$.

CHIU, Y.; SHIH, H.; WANG, C. 2012. Management tactics and investigation on insecticide resistance of chilli thrips on mango. Formosan Entomologist Special 15: 221-232. 
CHUN-NAN, L.; MIAO-YING, W.; NIANN-TAI, C.; YUAN, C. 2015. The occurrence of Scirtothrips dorsalis Hood in mango orchards and factors influencing its population dynamics in Taiwan. Asia-Pacific Entomology 18: 361-367.

DE LA ROSA, J. 2010. Abundancia y distribución espacio-temporal de trips asociados a flores de mango. Tesis de maestría en ciencias en recursos naturales y desarrollo rural. Colegio de la Frontera del Sur, Chiapas, México. 48 p.

DUARTE DE OLIVEIRA, M.; RABELO, F.; ALMEIDA-CORTEZ, J.; PATERNO, L. 2011. Espécies de Frankliniella (Thysanoptera, Thripidae): novos registros em mangueira (Mangifera indica) no Brasil. Ciencia Rural 41: 1709-1711.

DURÁN, Y. 2012. Evaluación de insecticidas para el control de plagas en mango (Mangifera indica L.) en Tierra Caliente, Guerrero, México. Tesis de Maestría en Ciencias, fitosanidad, entomología y acarología. Colegio de postgrados, Institución de enseñanza e investigación en ciencias agrícolas, Montecillo. $39 \mathrm{p}$.

FAO, Organización de las Naciones Unidas para la Alimentación y la Agricultura. 2012. La producción mundial de fruta tropical alcanzará 82 millones de toneladas en 2014. Disponible en: $\mathrm{https}$ ://theearthsfund.com/la-produccion-mundial-de-fruta-tropical-alcanzara-82-millones-de-toneladas-en-2014/. [Fecha revisión: 05 mayo 2016].

GARCÍA, J. 2011. Fenología del cultivo del mango (Mangifera indica L.) en el alto y bajo Magdalena: bases conceptuales para su manipulación. Corpoica, Editorial Produmedios; $1^{\text {era }}$ edición. $76 \mathrm{p}$.

GARCIA, P. 2012. Control químico en mango (Mangifera indica L.) contra escama blanca y trips en Tierra Blanca, Veracruz, México. Tesis de maestría en Ciencias, fitosanidad, entomología y acarología. Colegio de postgrados, Institución de enseñanza e investigación en ciencias agrícolas, Montecillo. $42 \mathrm{p}$.

GARCÍA-ESCAMILLA, P.; DURÁN-TRUJILLO, Y.; LÁZARODZUL, M.; VARGAS-MADRÍZ, H.; ACUÑA-SOTO, J. 2016. Manejo de trips (Frankliniella spp.) en mango (Mangifera indi$c a$ L.) a base de azufre en Veracruz, México. Entomología Agrícola 3: 441-444.

GIL V., L. F.; ARCILA C., A. M.; ACHURY M., R. A; SANABRIA B., M. C.; ARIAS B., H.; BAQUERO L., K. Y. 2013. Guía de campo para la identificación y manejo de enfermedades y plagas en el cultivo de mango. Disponible en: https://sioc.minagricultura.gov.co/Mango/Documentos/002\%20-\%20Cifras\%20Sectoriales $/ 002 \% 20-20$ Cifras $\% 20$ Sectoriales $\% 20-\% 202018 \% 20$ Marzo\%20Mango.pdf. [Fecha revisión: 13 septiembre 2018].

LEITE, G.; PICANÇO, M.; ZANUNCIO, J.; ECOLE, C. 2006. Factors affecting herbivory of thrips palmi (Thysanoptera: Thripidae) and Aphis gossypii (Homoptera: Aphididae) on the eggplant (Solanum melongena). Brazilian Archives of Biology and Technology 49: 361-369.

LOPES, R.; TAMAI, M.; BATISTA, S.; SILVEIRA, S.; DE SALVO, S. 2002. Occurrence of thrips on Niagara table grape and its control with the insecticides thiacloprid and methiocarb associated with Metarhizium anisopliae. Revista Brasileira de Fruticultura 24 (3): 269-272.

LÓPEZ-GUILLÉN, G.; ROSA-CANCINO, J.; GOLDARAZENA, A. 2014. Abundancia y fluctuación poblacional de trips asociados a hojas de mango Ataulfo en el Soconusco, Chiapas. Entomología Mexicana 1: 824-828.

MONTEIRO, R.; MOUND, L.; ZUCCHI, R. 2001. Espécies de Frankliniella (Thysanoptera: Thripidae) de importância agrícola no Brasil. Neotropical Entomology 30 (1): 65-72.

MUNJ, A.; JALGAONKAR, V.; SALVI, B.; NARANGALKAR, A. 2012. Seasonal incidence and control of mango thrips. Pestology 36 (12): 35-37.

ORTIZ, J.; INFANTE, F.; ZABALA, J. 2016. Ciclo de vida en laboratorio y sitios de oviposición de Frankliniella invasor Sakimura 1972 (Thysanoptera: Thripidae) en panículas de mango Ataulfo. Entomología Mexicana 3: 420-424.
R PROJECT. 2016. R versión 3.3.2, "Sincere Pumpkin Patch" Copyright (C). The R Foundation for Statistical Computing Platform: i386-w64-mingw32/i386 (32-bit).

RETANA-SALAZAR, A.; RODRÍGUEZ-ARRIETA, J. 2015. Especies potenciales de thrips (Insecta: Thysanoptera: Thripidae) en la polinización del mango (Mangifera indica L.) en Costa Rica. Revista Gaditana de Entomología. 6 (1): 103-112.

ROCHA, F.; INFANTE, F.; QUILANTAN, J.; GOLDARAZENA, A.; FUNDERBURK, J. 2012. 'Ataulfo' mango flowers contain a diversity of thrips (Thysanoptera). BioOne Research Evolved 95 (1): 171-178.

ROCHA, F.; INFANTE, F.; CASTILLO, A.; IBARRA-NUÑEZ, G.; FUNDERBURK, J. 2015. Natural enemies of the Frankliniella complex species (Thysanoptera: Thripidae) in Ataulfo mango Agroecosystems. Insect Science 15 (1): 114.

SALAZAR, M. 2012. Identificación, distribución y dinámica poblacional de escamas, trips y ácaros en mango (Mangifera indica L.) en Veracruz, México. Tesis de maestría en ciencias. Colegio de postgrados, Institución de enseñanza e investigación en ciencias agrícolas, Montecillo. $82 \mathrm{p}$.

SANTOS, O. 2010. Determinación del nivel de daño económico y la fluctuación poblacional de Neohydato thrips signifer (Priesner 1932). (Thysanoptera: Thripidae) en maracuyá (Passiflora edulis Degener) var. flavicarpa en el municipio de Suaza (Huila). Trabajo de grado de ingeniero agrónomo. Universidad Nacional de Colombia. $59 \mathrm{p}$.

SAS. 2009. SAS Institute, Inc, user guide: Statistical Analysis System, version 9.0. Cary, NC, EE. UU.

SIERRA, P. 2017. Nivel de daño económico y fluctuación poblacional de trips (Frankliniella cf. gardeniae Moulton) en mango en Tolima, Colombia. Trabajo de grado de Magíster en Ciencias Biológicas. Universidad de Caldas. Manizales, Colombia. 56 p.

SIOC. 2018. Sistema de información de gestión y desempeño de organizaciones de cadenas. 002 cifras sectoriales- 2018 marzo mango. Disponible en: https://sioc.minagricultura.gov.co/ Mango/Documentos/002 \%20- \%20Cifras \%20Sectoriales/002 $\% 20$ - \%20Cifras \%20Sectoriales \%20- \%202018\%20Marzo \% 20Mango.pdf. [Fecha revisión: 25 de agosto de 2018].

SOLÍS, P. 2016. Plan de manejo de trips en el cultivo de aguacate hass. INTA Costa Rica. Disponible en: http://repiica.iica.int/ docs/B4226e/B4226e.pdf. [Fecha de revisión: 17 octubre 2018].

SOTO-RODRÍGUEZ, G.; NISHIDA, K.; RETANA-SALAZAR, A. 2012. Un género nuevo de tisanóptero morador de agallas en Costa Rica (Thysanoptera: Phlaeothripidae: Phlaeothripinae). Revista Mexicana de Biodiversidad 83 (3): 605-610.

URÍAS-LOPEZ, M.; SALAZAR-GARCÍA, S.; JOHANSEN-NAIME, R. 2007. Identificación y fluctuación poblacional de especies de trips (Thysanoptera) en aguacate 'Hass' en Nayarit, México. Revista Chapingo Serie Horticultura 13 (1): 49-54.

WANG, J.; TONG, X. 2012. Species diversity, seasonal dynamics, and vertical distribution of litter-dwelling thrips in an urban forest remnant of South China. Journal of Insect Science 12 (67): $1-12$.

YADAV, R.; CHANG, N.-T. 2014. Effects of temperature on the development and population growth of the melon thrips, Thrips palmi, on eggplant, Solanum melongena. Journal of Insect Science 14 (78): 1-9.

Recibido: 25-sep-2017 • Aceptado: 25-oct-2018

Citación sugerida:

SIERRA-BAQUERO, P. V.; VARÓN-DEVIA, E. H.; GOMESDÍAS, L.; JARAMILLO-BARRIOS, C. I. 2018. Fluctuación poblacional de trips (Frankliniella cf. gardeniae) en cultivos de mango en Tolima, Colombia. Revista Colombiana de Entomología 44 (2): 158-164. Julio - Diciembre 2018. 